\title{
Quand science \\ et musique \\ se rencontrent
}

Garance Alberman ${ }^{1}$, Jean-Marie Gagez ${ }^{2}$,

Judith Miné-Hattab ${ }^{3}$

> Musique et sciences ont souvent été liées dans

l'histoire de la musique. Cependant, rares sont les compositeurs qui ont eu l'occasion d'interagir avec un scientifique pour s'imprégner de son travail et l'utiliser comme source d'inspiration. Le projet Muse-IC se propose de donner cette opportunité à des compositeurs d'aujourd'hui, en leur commandant une œuvre inspirée par une découverte scientifique récente. À la suite d'un double appel réalisé auprès de chercheurs et de compositeurs, des compositeurs se sont immergés dans l'univers scientifique de chercheurs afin de repousser les limites de leur démarche créative. Entre 2017 et 2019, six compositeurs ont écrit une pièce inspirée de la découverte d'un chercheur avec lequel ils ont interagit directement. L'aboutissement de ce projet a donné lieu à une conférence-concert à la salle Cortot, à Paris, une occasion unique de sensibiliser le public à l'importance de la recherche fondamentale au travers d'une rencontre originale entre compositeurs, musiciens et chercheurs. <

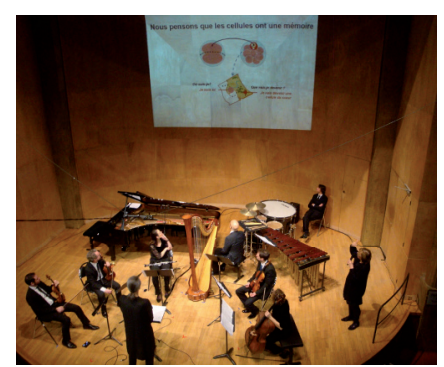

${ }^{1}$ Directrice artistique du projet Muse-IC, Paris, France.

${ }^{2}$ Compositeur, Paris, France.

${ }^{3}$ Institut Curie-PSL Research University, CNRS, Sorbonne Université, UMR3664, 26 rue d'UIm, 75005 Paris, France.

judith.mine@curie.fr

au contraire comme la réminiscence d'une tradition immémoriale dans laquelle la musique était elle-même considérée comme une science.

Incarnation de l'harmonie universelle, considérée comme « science sœur de l'astronomie » par Pythagore et Platon qui voyaient en elle le moyen de décrire les rapports harmoniques du cosmos, la musique sera enseignée comme science du nombre jusqu'à la Renaissance dans les arts libéraux du quadrivium, siégeant aux côtés de l'arithmétique, la géométrie et l'astronomie.

Mais si cette place de premier ordre - aux antipodes de notre vision actuelle d'une musique comme simple divertissement - se révèle capitale pour comprendre I'histoire de la musique, elle l'est également pour I'histoire des sciences. On oublie trop souvent que des scientifiques aussi importants que Oresme, Kepler, Galilée, Newton ou encore Herschel, ont aussi étudié la musique. Du côté des compositeurs, en revanche, malgré un intérêt certain tout au long de l'histoire, il faut reconnaître que ces initiatives, bien qu'intéressantes, sont souvent restées circonscrites dans leurs productions, touchant plus à la théorie qu'au langage musical lui-même.

Tout change au début $\mathrm{du} x \mathrm{x}^{\mathrm{e}}$ siècle lorsque, face à l'épuisement du système tonal qui régissait la musique occidentale depuis le xvII ${ }^{\mathrm{e}}$ siècle, cet intérêt des compositeurs pour la science va prendre une nouvelle dimension bien plus fondamentale et déterminante que par le passé. La crise du langage musical et la question de son renouvellement opèrent progressivement un changement de paradigme contraignant les compositeurs à explorer de nouvelles voies, à chercher de nouveaux modèles structurants, resserrant ainsi les liens entre 
composition et recherche scientifique. Utilisant les moyens d'investigation offerts par l'évolution de la technologie, nombre de musiciens deviennent de véritables chercheurs, situation nouvelle dont témoignera en France la création de l'Institut de recherche et coordination acoustique/musique (IRCAM), en 1970.

Parallèlement, l'accélération fulgurante des progrès scientifiques tout au long du siècle dernier jusqu'à aujourd'hui apparaît, dès lors, comme une source d'inspiration providentielle offrant aux musiciens de nouvelles voies d'investigation. Celles-ci s'élargissent d'autant plus qu'aux rapports traditionnels avec la physique, les mathématiques et l'astronomie, viennent désormais s'ajouter les nouvelles perspectives offertes par les découvertes de la biologie.

Pour les compositeurs, que ce soit dans la forme globale de l'œuvre, dans le traitement du matériau musical ou dans la définition du geste instrumental, ces interactions, plus structurelles que par le passé, ne cessent d'élargir le champ de la création musicale en variant leurs modes d'appréhension du phénomène sonore : il ne s'agit plus seulement d'évocations poétiques et de rapports symboliques au sein d'un système d'écriture tonal possédant sa propre logique, mais d'opérer un renouvellement du matériau musical au sein de structures originales élaborées à partir de modèles extérieurs à la musique via la conversion de données ou la création d'analogies conceptuelles. Du côté de la science, face à une réalité qui se révèle toujours plus complexe à mesure qu'on l'étudie, cette dynamique de convergence donne également aux scientifiques la possibilité d'enrichir leur pratique en se confrontant à une appréhension différente de leur domaine de recherche.

C'est dans cette perspective que s'inscrit le projet Muse-IC [1] initié en 2017. Né du désir de créer des musiques originales en symbiose avec des découvertes scientifiques récentes, il se concrétise grâce à l'aide financière de l'Université Paris Sciences et Lettre ${ }^{1}$. L'Institut Curie hébergera le projet, ce qui lui donnera son nom, «Muse-IC». II participera au financement du concert final en 2019. Son principe : favoriser la rencontre entre les musiciens, les scientifiques et le public en commandant à six compositeurs internationaux une œuvre de dix minutes inspirée par une découverte scientifique récente. Au cours du projet, les compositeurs ont pu choisir tout ou une partie de la nomenclature proposée: piano, quatuor à cordes, clarinette, harpe, percussion. Ils ont ensuite choisi le thème scientifique qui les inspirait le plus parmi une quinzaine de découvertes préalablement sélectionnées par Judith Miné-Hattab, après un appel réalisé auprès de scientifiques motivés par le projet. Durant leur processus de création, les compositeurs ont pu interagir avec les chercheurs concernés au travers de discussions et de visites de laboratoires afin d'appréhender leur sujet en profondeur. Pour le chercheur, Muse-IC est une occasion rêvée de partager la beauté de sa découverte avec un public non scientifique au travers de la musique. Pour le compositeur, s'imprégner d'un sujet inconnu en pénétrant dans le monde des laboratoires, est une occasion unique de stimuler sa démarche créative, d'élargir son champ d'inspiration et parfois de développer de nouvelles techniques de compositions. Chaque œuvre composée est née d'un échange entre un chercheur et un compo-

\footnotetext{
${ }^{1}$ Appel d'offre « Aux Frontières des Labex » 2015.
}

siteur. Les sujets proposés ont été volontairement choisis de manières diverses avec néanmoins un accent mis sur la biologie, un domaine très peu illustré en musique. Au fil des mois, confronter ces deux univers s'est avéré une expérience fascinante: entre discussions et visite de laboratoires, un dialogue naturel et efficace s'est installé entre chaque «duo chercheur-compositeur».

Aboutissement du projet, le concert du 12 mars 2019 à la salle Cortot (Paris), organisé par Garance Alberman, a permis de faire entendre au public le fruit de ces interactions. Chaque œuvre était précédée d'une courte présentation scientifique destinée à plonger les auditeurs dans le contexte de l'œuvre (Figure 1). Les œuvres, magnifiquement interprétées par huit musiciens de l'Orchestre philharmonique de Radio France et l'Orchestre national de France, sous la direction de Marc Desmons (premier chef invité de l'ensemble $\left.T M_{+}\right)^{2}$, ont été enregistrées lors du concert ${ }^{3}$. Un documentaire ${ }^{4}$, réalisé par Frédéric Dano, relate également les différents projets scientifiques illustrés en musique ainsi que les échanges entre scientifiques et compositeurs au cours de ce projet.

\section{Les œuvres composées pour le projet Muse-IC}

Du déroulement multiple et révélé du temps, affleure le bal des retrouvailles, Emmanuel Hieaux

C'est sur un accord arpégé de piano que s'est ouvert le concert. En ouverture, Emmanuel Hieaux nous propose une pièce intitulée $D u$ déroulement multiple et révélé $d u$ temps, affleure le bal des retrouvailles, inspirée des travaux de Judith Miné-Hattab et al. (Institut Curie) sur la dynamique multiéchelles de la chromatine, en réponse aux dommages de l'ADN $[2-5](\rightarrow)$.

Cette pièce, écrite pour un $(\rightarrow)$ Voir les Nouvelles de J. Miné-Hattab et R. Rothstein, $m / s n^{\circ} 8-9$, page 714 , et J. MinéHattab et X. Darzacq, $\mathrm{m} / \mathrm{s} \mathrm{n}^{\circ} 10$, octobre 2018 , page 778 août-septembre 2012, ensemble d'instruments original (clarinette en La, quatuor à cordes, marimba et piano) nous invite à découvrir comment I'ADN long de 2 mètres s'enroule pour pénétrer dans le noyau cellulaire, puis comment les cassures de I'ADN affectent son mouvement. Emmanuel Hieaux perçoit dans ce sujet «une allégorie à la vie dont la mise en musique allait lui permettre d'unir la description rigoureuse de la découverte à une harmonieuse licence poétique ». Sous son prisme, la dynamique de I'ADN devient un conte poétique, une pièce en 3 mouvements :

${ }^{2}$ TM+ est un ensemble de musique contemporaine français créé en 1986 et dirigé par Laurent Cuniot. II est en résidence à la Maison de la musique de Nanterre depuis 1996. ${ }^{3}$ https://curie.fr/actualite/evenement/muse-ic-quand-science-et-musique-serencontrent

${ }^{4}$ https://www.youtube.com/watch?v=Rd20IFFXiCk\&t=693s 


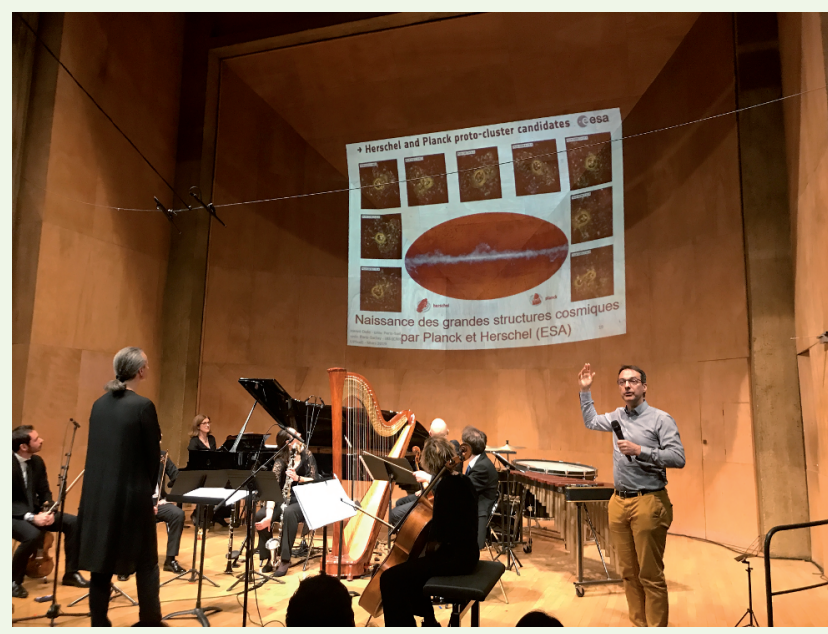

Figure 1. Hervé Dole (astrophysicien à l'Institut d'astrophysique spatiale d'Orsay) présente ses travaux avant l'interprétation des pièces de Geoffrey Gordon et de Jean-Marie Gagez inspirées du sujet proposé. Sur scène, de gauche à droite : Cyril Baleton (violon), Marc Desmons (direction), Joseph André (violon), Catherine Cournot (piano), Christelle Pochet (clarinette), Nicolas Tulliez (harpe), Julien Dabonneville (alto) et Pauline Bartissol (violoncelle) (photographie (c) Frédéric Dano, réalisée lors du concert le 12 mars 2019, Salle Cortot, Paris).

《insouciances, rupture et réparation $»^{5}$. «L'ADN double-brin est interprété par l'alto et la clarinette dont les sons longs et legato symbolisent le long double brin. Indissociables l'un de l'autre, ils ne cesseront de dialoguer, toujours unis, que ce soit dans la sérénité, l'insouciance, le désir, la douleur, la souffrance et la paix retrouvée. Les histones, autour desquelles s'enroule le double-brin qui sont autant d'entités indépendantes l'une de l'autre; le piano, le marimba, le violoncelle et les deux violons chanteront, danseront en un contrepoint mélodique et rythmique. Un court prélude laisse résonner les arpèges du piano. Puis, dans un univers peuplé d'entités qui se meuvent dans une parfaite plénitude, un long double-brin d'ADN apparaît, séduit et mu par un désir sans cesse grandissant; il s'enroule autour d'elles, se resserre et devient une minuscule et dense pelote qui, dans un rythme de plus en plus effréné, danse audessus du volcan. Survient la cassure. Le double-brin fractionné s'affole et souffre d'avoir perdu une partie de lui-même. S'ensuit le temps de la réparation. Il lui faut retrouver son double qui lui remettra la partie manquante et lui permettra de retrouver son unité. Une fois cela accompli, revient la paix, et l'œuvre s'achève dans un postlude serein ».

\section{Quand I'ADN fait des boucles, Amir Bitran}

L'enroulement et la dynamique complexe de la molécule d'ADN à l'intérieur de nos cellules est également à l'origine de la pièce composée par Amir Bitran (scientifique à Harvard University, Boston, États-Unis, et compositeur) inspiré d'un sujet proposé par Léonid Mirny (Massachu${ }^{5}$ Les textes marqués entre guillemets décrivant les œuvres composées sont tirés du programme du concert
du 12 mars 2019 et ont été écrits par les compositeurs. setts Institute of Technology, Cambridge, États-Unis). L'équipe de Leonid Mirny étudie l'organisation tri-dimensionnelle de la molécule d'ADN, en particulier, les différents niveaux de structures qui forment et hiérarchisent notre génome. Il a récemment proposé un mécanisme simple pour expliquer la formation de la structure de la chromatine à l'échelle de quelques kilobases $[6,7]$. Notamment, la chromatine, à l'intérieur d'un domaine, serait organisée en boucles dynamiques: celles-ci grandiraient de plus en plus grâce à des protéines, permettant ainsi le rapprochement de régions éloignées du génome. La pièce composée par Amir Bitran est une peinture musicale de l'ADN de notre corps et de ses dynamiques et structures changeantes et élégantes à la fois.

"Sur le plan musical, ces boucles émergent tout d'abord de l'alto et sont ensuite transmises à d'autres instruments; elles grandissent et se rétractent pour créer des textures plus élaborées évoquant l'action et l'organisation concertées de multiples boucles dans I'ADN. Mais outre ce processus hautement dirigé et guidé, notre génome est également sculpté par des forces plus passives s'apparentant à la gravité, qui provoquent l'effondrement et l'union de différentes parties de l'ADN. Cet effondrement est décrit musicalement quelques minutes après le début de l'œuvre par des glissandi descendants ou encore des sonorités ressemblant à des soupirs. Au fur et à mesure que la pièce avance, on parvient à distinguer un certain rapprochement entre ces différentes textures musicales, qui finissent par se transformer en un grand final rappelant un choral. Les forces qui organisent notre génome à l'échelle microscopique sont changeantes et chaotiques, mais elles créent collectivement de magnifiques structures qui génèrent l'incroyable diversité de la vie sur Terre ».

\section{Quiescence, Alexandra du Bois}

Si les secrets de la molécule d'ADN ont inspiré les deux pièces précédentes, l'œuvre suivante est inspirée par une découverte récente de biologie cellulaire. Quiescence, composée par Alexandra du Bois pour clarinette basse, violon, alto, violoncelle et piano, s'inspire du sujet "Quand nos cellules sommeillent: quiescence et renaissance» proposé par l'équipe d'Angela Taddei (Institut Curie). En l'absence d'un ou plusieurs nutriments, les cellules vivantes ont la capacité étonnante de pouvoir entrer dans un état alternatif «dormant», appelé «quiescence». Issue du terme «quiétude», la quiescence est un état dans lequel la cellule sommeille: son activité transcriptionnelle est très fortement diminuée. Elle reste alors viable pendant une période très longue (parfois plusieurs mois) sans aucun apport nutritif. L'organisation du noyau est alors tota- 
lement bouleversée : chez la levure, les télomères se réorganisent de façon spectaculaire en se regroupant sous forme d'un hyper-cluster situé au centre du noyau [8] ; autour de cet hyper-cluster, la chromatine devient extrêmement dense en suivant une compaction concentrique allant du centre à la périphérie du noyau. Après s'être nourrie des substances nutritives manquantes, la cellule renait et le noyau retrouve son organisation initiale en moins de 30 minutes.

Dans le quintette Quiescence, la clarinette basse évoque la quiescence, le calme, la densité, la profondeur, puis l'intensité du violon évoque la renaissance de la cellule lors du réveil de quiescence. Le piano reflète les clignotements de la technique de microscopie super résolution PALM ${ }^{6}$ utilisée pour mesurer la compaction de la chromatine.

\section{Aion, Denis Ramos}

De la cellule vers un organisme entier et la biologie du développement, la pièce suivante, Aion, composée par Denis Ramos, s'inspire du sujet De la cellule à l'embryon: ces heures cruciales qui nous façonnent, proposé par l'équipe de Nathalie Dostatni. Au cours du processus de développement, les cellules savent exactement où elles se trouvent et ce qu'elles doivent devenir pour former l'organisme. L'équipe de Nathalie Dostatni étudie la phase précoce du développement de l'embryon de drosophile, en particulier comment l'embryon connaît I'orientation de son corps : où se trouveront le haut, le bas, la gauche, la droite, le devant et l'arrière du corps. Selon Nathalie Dostatni, les cellules auraient une mémoire leur permettant de savoir qui était leur mère, et de se souvenir des instructions transmises, de sorte qu'elles savent où elles se trouvent et ce qu'elles deviendront $[9,10]$.

Au travers des films de microscopie réalisés par l'équipe, Denis Ramos perçoit «le temps cyclique qui se répète et l'idée d'éternité ». II traduit cela dans sa pièce intitulée Aion, une divinité primordiale dans la mythologie grecque, ainsi qu'un concept philosophique antique lié à l'infinitude du temps. Ce mot signifie littéralement «durée de la vie », puis par extension « destinée » et «éternité ». Dans sa partition, Denis Ramos a développé « une polyphonie musicale qui se veut le reflet des processus de division et de prolifération cellulaires. La notion de cycle $y$ est fondamentale. Le flux polyphonique se répartit en trois groupes instrumentaux: le quatuor à cordes, le duo clarinette/marimba, et la harpe. Un préambule et un postambule encadrent et complètent ce long processus sonore qui se déploie durant près de huit minutes ».

\section{Les Voies de la Lumière, Jean-Marie Gagez}

Seule pièce du concert écrite pour un instrument seul, Les Voies de la Lumière de Jean-Marie Gagez, s'inspire du sujet Les premières vibrations de l'univers et la quête des premiers amas de galaxies proposé par l'astrophysicien Hervé Dole de l'Institut d'astrophysique spatiale, à Orsay. Ce vaste sujet représente un travail de grande envergure développé au

\footnotetext{
${ }^{6}$ PALM (photo-activated localization micrsocopy) est une technique de microscopie super résolution récemment développée permettant de visualiser chaque molécule présente dans des objets biologiques avec une précision allant jusqu'à 20 nanomètres. On parle de microscopie à l'échelle de la molécule unique. Le principe de cette technique est de faire apparaitre chaque molécule l'une après l'autre, de manière à pouvoir les localiser au-delà de la limite de la diffraction de la lumière. Ces expériences, dans lesquelles chaque molécule brille puis s'éteint l'une après l'autre, révèlent la structure complexe et détaillée d'un objet biologique dans un rendu très visuel et poétique.
}

sein du programme collaboratif Planck $[11,12]$ et rendu accessible à un public non spécialiste dans l'ouvrage « Le côté obscur de l'univers » [13]. Jusqu'en 380000 ans après le big bang, le cosmos apparaît dense et opaque, le mouvement des photons étant entravé par la jungle touffue des électrons libres. Mais à cette date, sous la dilution et le refroidissement de l'univers, les électrons perdent leur liberté et se retrouvent confinés dans des atomes: I'univers devient transparent, laissant ainsi apparaître la lumière du rayonnement fossile. Le cosmos s'illumine alors, mais cette lumière est diffuse car les sources de lumière localisées que sont les étoiles et les galaxies n'ont pas encore fait leur apparition. Mesurer avec une précision sans précédent grâce au lancement du satellite Planck en 2009 les propriétés de la lumière du rayonnement fossile, appelée également fond diffus cosmologique, forme une sorte d'écho lumineux du Big bang traduisant ses premières vibrations. Cette lumière apporte un éclairage nouveau sur deux périodes particulièrement énigmatiques : I'inflation juste après le big bang et la sortie des âges sombres quand les premières étoiles et galaxies se sont formées. En effet, sous l'effet de son expansion, l'univers se dilue et se refroidit entraînant l'inversion du rapport énergétique entre lumière et matière. La lumière va peu à peu perdre de sa dominance et c'est la matière qui va prendre le contrôle des affaires de l'univers. La gravité va faire croître les semences de galaxies, détectées comme de minuscules fluctuations de température du rayonnement fossile. Des structures de plus en plus élaborées font leur apparition au cours du temps jusqu'à ce que les boules gazeuses formant les étoiles s'allument, marquant ainsi la fin de l'ère préstellaire.

Dans Les Voies de la Lumière, le paradoxe entre l'immensité du sujet et le choix d'un unique instrument permet au compositeur Jean-Marie Gagez de recréer sur scène l'impression d'espace tout en exprimant l'unité primordiale du fond diffus cosmologique. Dans la pénombre spécialement créée pour l'occasion, le compositeur travaille tout d'abord sur l'idée de transparence en faisant naître du silence d'amples respirations dont les vibrations s'enrichissent progressivement d'harmoniques jusqu'à saturation. Une seconde phase met en évidence le découpage harmonique du cosmos duquel naît une lente méditation plus mélodique et rythmique, évoquant la mise en place des premiers amas de galaxies. Clin d'œil à la théorie des cordes, seules les quatre cordes à vide de l'instrument et leurs harmoniques naturelles sont utilisées, chaque corde représentant symboliquement l'une des quatre forces fondamentales qui régissent l'univers (gravitationnelle, électromagnétique, interaction faible et interaction forte). 


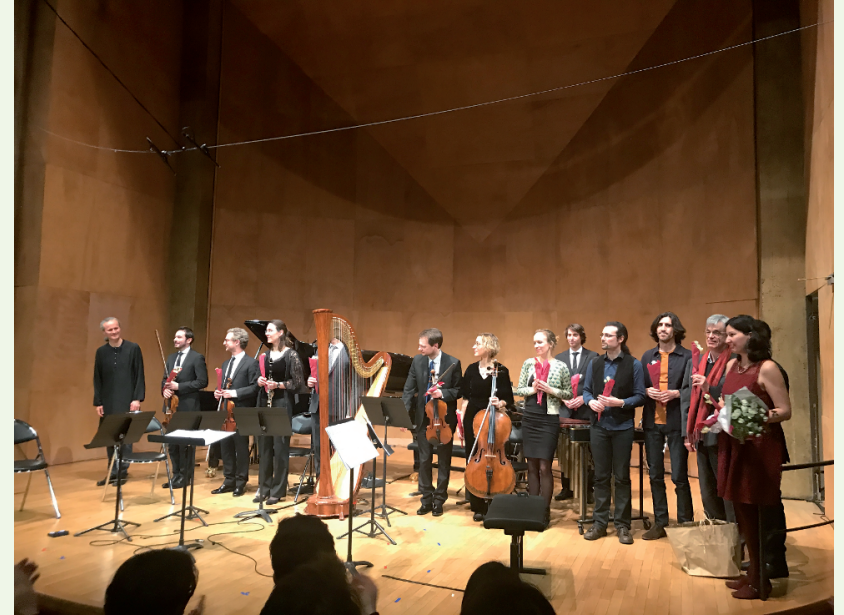

Figure 2. Salut final lors du concert du 12 mars 2019 Salle Cortot, Paris. De gauche à droite: Marc Desmons, Cyril Baleton, Joseph André, Christelle Pochet, Nicolas Tulliez, Julien Dabonneville, Pauline Bartissol, Alexandra du Bois, Gabriel Benlolo, Jean-Marie Gagez, Denis Ramos, Emmanuel Hieaux, Amir Bitran et Judith Miné-Hattab (@ Frédéric Dano).

Inflation cosmique et la fin des âges sombres, Geoffrey Gordon Également inspirée du sujet proposé par Hervé Dole, la pièce de Geoffrey Gordon contraste sur tous les points avec la composition de JeanMarie Gagez. Composée pour clarinette, harpe, piano et quatuor à cordes, «Inflation cosmique et la fin des âges sombres » exprime «la violence et la transparence de ces moments intenses de création». Geoffrey Gordon a développé dans sa pièce des effets sonores et des timbres originaux mêlant sonorités qui semblent en mouvement, harmoniques limpides, pizzicati aux cordes, glissandi de harpe intermittents et gémissements profonds de la clarinette basse. En dix minutes concises, la musique de ce moment extraordinaire de la vie de l'univers est exprimée et explorée.

\section{Conclusion}

Montrant une fois de plus la pertinence et la richesse des interactions réciproques entre science et musique, le concert du 12 mars 2019 a consacré cette volonté de rapprochement et d'échange entre scientifiques, compositeurs et public (Figure 2). Ce dernier ne s'y est pas trompé. Venus nombreux ce soir-là, les auditeurs se sont montrés particulièrement réceptifs et enthousiastes à ces approches différentes mais complémentaires offrant une porte d'accès à la fois originale et pédagogique vers la recherche scientifique de pointe et la musique contemporaine. Les perspectives ouvertes par la mise en valeur de l'interdisciplinarité dans la recherche et le désir des différents acteurs du projet d'inscrire cette démarche dans la durée élargissent le champ des possibles en laissant présager d'une suite dont les modalités restent encore à définir. $\diamond$

When science and music meet

\section{REMERCIEMENTS}

Nous remercions Emmanuel Hieaux, Denis Ramos, Jean-Marie Gagez, Geoffrey Gordon, Amir Bitran, Alexandra duBois, Angela Taddei, Nathalie Dostatni, Leonid Mirny, Hervé Dole pour leur contribution tout au long du projet. Nous remercions également Fanny Gascuel, Léa Wurgess et Chloé Dupuis pour leur aide administrative.

Ce projet a été réalisé dans le cadre de l'Idex portant la référence ANR-10IDEX-0001-02-PSL. Nous tenons à remercier l'Université Paris Sciences et Lettres, le labex Deep, le laboratoire de Leonid Mirny ainsi que l'UMR 3664 Dynamique du Noyau pour leur soutien financier.

\section{LIENS D'INTÉRÊT}

Les auteurs déclarent n'avoir aucun lien d'intérêt concernant les données publiées dans cet article.

\section{RÉFÉRENCES}

1. Dolgin $\varepsilon$. The sounds of science: biochemistry and the cosmos inspire new music. Nature 2019 ; 569 : 190-1.

2. Miné-Hattab J, Rothstein R. Increased chromosome mobility facilitates homology search during recombination. Nat Cell Biol $2012 ; 14: 510-7$.

3. Miné-Hattab J, Recamier V, Izeddin I, et al. Multi-scale tracking reveals scale-dependent chromatin dynamics after DNA damage. Mol Biol Cell 2017. pii: mbc.E17-05-0317.

4. Miné-Hattab J, Rothstein R. Réparation de l'ADN : comment trouver le bon partenaire? Med Sci (Paris) $2012 ; 28: 714-6$.

5. Miné-Hattab J, Darzacq X. Dynamique de la chromatine en réponse aux dommages de l'ADN : une histoire multiéchelle. Med Sci (Paris) 2018 ; 34 ; 778-81

6. Fudenberg G, Imakaev M, Lu C, et al. Formation of chromosomal domains by loop extrusion. Cell Rep 2016 ; 15 : 2038-49.

7. Goloborodko A, Marko JF, Mirny LA. Chromosome compaction by active loop extrusion. Biophys J $2016 ; 110: 2162-8$.

8. Guidi M, Ruault M, Marbouty M, et al. Spatial reorganization of telomeres in long-lived quiescent cells. Genome Biol $2015 ; 16: 206$.

9. Lucas T, Ferraro T, Roelens B, et al. Live imaging of bicoid-dependent transcription in Drosophila embryos. Curr Biol 2013 ; 23 : 2135-9.

10. Lucas T, Tran H, Perez Romero CA, et al. 3 minutes to precisely measure morphogen concentration. PLoS Genet 2018; 14 : el007676.

11. Ade PAR, Aghanim N, Arnaud M, et al. Planck 2015 results. XIII. Cosmological parameters. Astronomy Astrophysics 2016 ; 594 : Al3.

12. Aghanim N, Altieri B, Arnaud M, et al. Planck intermediate results. XXVII. High-redshift infrared galaxy overdensity candidates and lensed sources discovered by Planck and confirmed by Herschel-SPIRE. Astronomy Astrophysics $2015 ; 582$ : A30.

13. Dole H. Le côté obscur de l'univers. Paris : Dunod, 2017 : 200 p.

\section{TIRÉS À PART}

J. Miné-Hattab
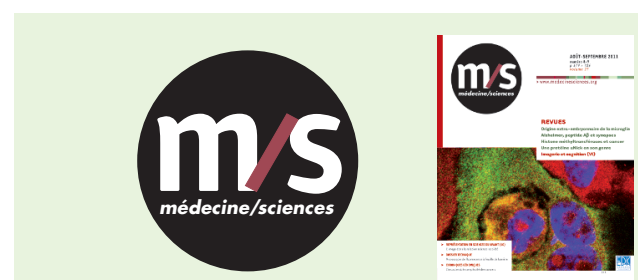

Abonnez-vous à médecine/sciences

Bulletin d'abonnement page 910 dans ce numéro de $\mathrm{m} / \mathrm{s}$ 